\title{
Harmoniser les pratiques éditoriales numériques des revues françaises d'archéologie
}

Harmonising Digital Editorial Practices in French Archaeological Journals

Stéphane Renault, Blandine Nouvel, Micaël Allainguillaume, Astrid Aschehoug, Nicolas Coquet et Marie-Adèle Turkovics

\section{(2) OpenEdition}

\section{Journals}

Édition électronique

URL : https://journals.openedition.org/revuehn/483

DOI : 10.4000/revuehn.483

ISSN : 2736-2337

Éditeur

Humanistica

\section{Référence électronique}

Stéphane Renault, Blandine Nouvel, Micaël Allainguillaume, Astrid Aschehoug, Nicolas Coquet et Marie-Adèle Turkovics, « Harmoniser les pratiques éditoriales numériques des revues françaises d'archéologie », Humanités numériques [En ligne], 2 | 2020, mis en ligne le 01 juin 2020, consulté le 15 juillet 2021. URL : http://journals.openedition.org/revuehn/483 ; DOI : https://doi.org/10.4000/ revuehn. 483

Les contenus de la revue Humanités numériques sont mis à disposition selon les termes de la Licence Creative Commons Attribution 4.0 International. 


\section{humanités \\ numériques}

\section{Harmoniser les pratiques éditoriales numériques des revues françaises d'archéologie}

\section{Harmonising Digital Editorial Practices in French Archaeological Journals}

Stéphane Renault, Blandine Nouvel, Micaël Allainguillaume, Astrid Aschehoug, Nicolas Coquet et Marie-Adèle Turkovics

\section{Résumés}

Cet article présente un projet d'harmonisation de la politique d'édition numérique de cinq revues d'archéologie qui s'inscrivent dans un contexte concernant une quarantaine de supports éditoriaux du domaine en France. Mettant à profit des outils technologiques et documentaires disponibles pour l'édition, en archéologie et dans ses sciences connexes en particulier, ces revues ont décidé de faire évoluer leurs pratiques en adoptant une grammaire et un corpus de mots-clés communs et en expérimentant de nouveaux modèles interopérables de structuration et d'indexation de leurs contenus. Elles sont ainsi imbriquées dans une chaîne de production scientifique qui mêle volontiers référencement des publications et des données. La visée est d'élargir et de faciliter l'accès à l'information scientifique. 
This article presents a project to harmonise the digital publishing policies of five archaeological journals in a context involving about forty editorial supports in the field in France. Taking advantage of the technological and documentary tools available for publishing, especially in archaeology and its related sciences, these journals have decided to update their practices by adopting a shared grammar and a shared corpus of keywords and by experimenting with new interoperable models for structuring and indexing their contents. They are thus nested in a scientific production chain that readily references publication and data together. The aim is to widen and facilitate access to scientific information.

\section{Entrées d'index}

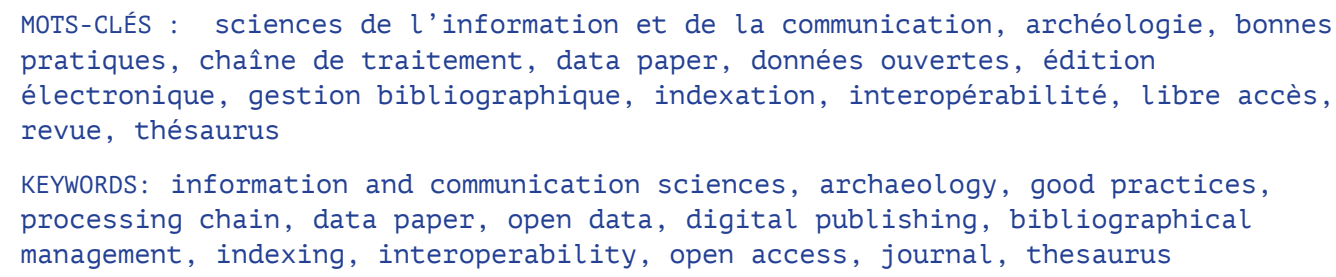

L’archéologie française dispose d'une cinquantaine de revues et de quasiment autant de collections pour publier le fruit de ses recherches. La multiplicité de ces supports génère naturellement une hétérogénéité des pratiques éditoriales. L'édition numérique, le développement d'outils adaptés et la mise en place d'infrastructures nationales d'édition, de diffusion et d'exposition des données de la recherche offrent un environnement favorable à la promotion de bonnes pratiques dont l'objectif est d'accroître la visibilité, le partage et la réutilisation des résultats de la discipline. Dans ce contexte de science ouverte et de développement technologique efficient, cinq revues d'archéologie harmonisent leurs pratiques en édition numérique en adoptant une grammaire et un corpus de mots-clés communs et en expérimentant de nouveaux modèles interopérables de structuration et d'indexation de leurs contenus. Après une présentation de la situation française pour les revues scientifiques en sciences humaines et sociales (SHS), et en particulier en archéologie, nous exposons les objectifs visés de cinq de ces revues qui mettent à profit un contexte scientifique et technique favorable à leur démarche. Si l'opération n'est pas encore tout à fait terminée, elle s'ouvre sur des perspectives encourageantes ${ }^{1}$. 


\section{Les revues françaises d'archéologie et le mouvement de la science ouverte}

\section{Une organisation hétérogène}

L'archéologie et ses disciplines associées portent de manière intrinsèque un caractère pluridisciplinaire. Cette richesse a permis notamment l'émergence d'un nombre important de supports de publication à portée (inter-)régionale et (inter-)nationale. Les revues d'archéologie avec les collections et la plupart des ouvrages en sciences humaines et sociales sont fréquemment élaborés dans un laboratoire de recherche, dont ils traduisent le plus souvent la couverture thématique et chronologique, puis ils sont édités par une maison d'édition académique ou nationale. Fruits de l'histoire de la discipline, les revues peuvent aussi parfois être éditées par des associations et des sociétés savantes qui en assurent la fabrication et la diffusion. Les presses universitaires livrent également des publications en archéologie, sous la forme de monographies au sein de collections dédiées ${ }^{2}$.

Ainsi, revues et bulletins, qui garantissent un maillage géographique et chronologique de la production scientifique, reflètent la réalité de la recherche archéologique, à la fois locale et internationale. Cela conduit, selon le regard qu'on y porte, soit à un émiettement, soit à une offre avantageusement diversifiée des supports de publication. Cette offre éditoriale a fait l'objet, depuis une douzaine d'années, d'enquêtes réalisées notamment par le ministère de la Culture (Aubin 2007, Boucharlat et Mandy 2014). Elles ont porté sur l'analyse d'une quinzaine de revues financées ou soutenues par le ministère, huit revues interrégionales et sept revues nationales, et ont concerné aussi bien les aspects quantitatifs que qualitatifs, la structuration des équipes éditoriales que les modes de financement, les aspects liés à leur fabrication jusqu'à leur diffusion. Si la publication numérique était alors en discussion pour toutes ces revues, seules quelques-unes en 2007 avaient déjà opté pour ce mode diffusion (Aubin 2007: 134).

Cette diversité révèle son lot d'hétérogénéité, dans les structures éditoriales, dans les thématiques représentées, dans les moyens financiers alloués, dans les capacités à prendre en compte l'évolution des métiers des professionnels de l'édition, dans les modes de distribution, etc. Dans le même temps, en 2012, une étude réalisée par le groupe de travail " Fonctions et métiers (thème : bonnes pratiques : nomenclature métiers) » du réseau Médici - réseau national des professionnels de l'édition scientifique publique, CNRS - (Daunois et Roussel 2012) a montré que la professionnalisation des revues était encore perfectible. Ainsi, sur un échantillon de 357 revues concernées par l'étude, plus des deux tiers ne respectaient pas les critères que l'on est en droit d'attendre d'une revue scientifique publique, c'est-à-dire la présence systématique d'un directeur ou d'une directrice de publication, un schéma d'organisation de l'équipe éditoriale et des workflows clairs et structurés (appel à communication, sélection, modification, diffusion, etc.). Les revues d'archéologie n'échappent pas à ce constat. 
Concernant les aspects financiers, plusieurs institutions nationales apportent leur soutien aux revues françaises en sciences humaines et sociales. L'Institut des sciences humaines et sociales (INSHS) du CNRS a mis en place depuis 2011 une politique d'aide à l'édition scientifique ${ }^{3}$ " aussi bien à destination des revues établies qu'à l'attention de nouvelles initiatives éditoriales qui émergent sur certaines thématiques ou autour d'objets interdisciplinaires ". Ces fondements sont abordés par Odile Contat et Didier Torny (2015) dans un article rappelant les caractéristiques de l'édition scientifique en SHS en France. En 2017, sur les 2000 revues en sciences humaines et sociales recensées (Archambault et Vignola-Gagné 2004), 157 revues, toutes disciplines du domaine confondues, ont bénéficié du soutien de l'INSHS (subventions, personnels, traduction) sur les 171 ayant déposé un dossier pour l'aide 2018-2019. Parmi les revues soutenues par le ministère de la Culture, une quinzaine concerne l'archéologie (Boucharlat et Mandy 2014). Les autres bénéficient de ressources propres ou de personnels mis à disposition par le CNRS ou par d'autres institutions publiques comme l'Institut national de la recherche archéologique préventive (INRAP).

\section{Aborder le tournant du numérique}

Depuis l'enquête de Gérard Aubin de 2007, la tendance s'est inversée et la plupart des revues d'archéologie ont mis en place une politique de diffusion numérique, profitant de l'existence de portails de diffusion académique $^{4}$. Ainsi, certaines ont choisi la plateforme ouverte Persée, qui met en ligne les fichiers PDF et le plein texte des versions imprimées. D'autres ont privilégié Cairn, plateforme de revues scientifiques en accès ouvert ou disponibles sur abonnement. D'autres encore se sont rapprochées de Revues.org, devenu récemment OpenEdition Journals, qui diffuse des versions HTML et dérivées des textes (PDF, EPUB), avec potentiellement l'adoption du modèle Freemium comme modèle économique original ${ }^{5}$. Certaines mixent deux solutions, la première pour le fonds rétrospectif sur Persée, la seconde pour la production courante sur OpenEdition Journals, aidées en cela par les accords de coopération entre les deux plateformes qui permettent le passage aisé de l'une à l'autre. Enfin, des initiatives institutionnelles ont conduit au développement de portails propres, par exemple le site internet des publications du Muséum national d'histoire naturelle ${ }^{6}$.

Mais au-delà de la diffusion en ligne des textes, le passage progressif des revues d'archéologie à l'édition numérique s'accompagne de nouveaux enjeux, notamment sur les modalités d'accès aux résultats de la recherche. Car pour jouer le jeu de la "Grande conversation » scientifique décrite par Jean-Claude Guédon (2014), les politiques éditoriales doivent s'adapter à cette mutation pour redéfinir leur position dans un panorama de l'édition scientifique qui change d'échelle. Le secteur est en effet de plus en plus concurrentiel, les questions d'accès, libre ou sous embargo, de rapidité de publication, de pérennité et de visibilité, de conditions de réutilisation sont autant de points que les structures éditoriales se doivent de prendre en compte. Ces différents aspects ont été depuis longtemps mis en avant et discutés par la communauté scientifique et les institutions de recherche ${ }^{7}$. 
En 2015, l'Association des éditeurs de la recherche et de l'enseignement supérieur (AEDRES) s'est vue confier par la direction générale pour la recherche et l'innovation du ministère de l'Enseignement supérieur et de la Recherche la mission (Henny 2015) de dresser " un état des lieux des activités éditoriales des établissements d'enseignement supérieur et de recherche, d'apporter des informations sur les relations entre édition publique et édition privée et de formuler des propositions pour améliorer ces relations et dynamiser les politiques de valorisation de la recherche " (Hoareau-Dodinau 2015).

S'il n'est pas lieu ici de retracer la genèse du mouvement du libre accès, il faut garder à l'esprit, en France, l'appel de Jussieu de 2017 pour la science ouverte et la bibliodiversité ${ }^{8}$, le cadre de la loi Axelle Lemaire, dite pour une République numérique (articles 11, 30 et 38), et noter la volonté politique tout récemment affirmée par le ministère de l'Enseignement supérieur et de la Recherche dans son "Plan national pour la science ouverte ${ }^{9}$ ".

L'accès aux données de la recherche se pose comme nouvel enjeu majeur, tant du point de vue politique pour l'intégrité et l'évaluation de la recherche que du point de vue scientifique pour leur partage, leur archivage et leur réutilisation, ce dont les réglementations nationales et internationales s'emparent désormais pleinement. L'édition scientifique se trouve naturellement au cœur de ce dispositif. Elle exploite et recherche des solutions pour de nouvelles formes d'éditorialisation des textes et des données. À l'exception de quelques rares expériences comme l'écriture logiciste, la publication archéologique française a proposé jusqu’à ces dernières années un accès aux données primaires sous plusieurs formes :

- directement intégrées à l'article : elles sont faciles à rechercher et à citer, mais aussi assez peu réutilisables car difficiles à extraire de l'article. Elles sont, de fait, tributaires de la politique de la maison d'édition ;

- associées à l'article en annexe (ou supplementary data) : le format des données est alors libéré des contraintes rédactionnelles de l'article et la liaison des fichiers à l'article assure la paternité des données. Ces dernières sont également tributaires de la politique de la maison d'édition et les formats des fichiers sont peu standardisés. Les données sont en définitive difficilement exploitables indépendamment de l'article ;

- indépendantes de l'article et possiblement décrites dans des articles de données (data papers) : les jeux de données ainsi constitués sont accessibles à partir d'entrepôts généralistes ou disciplinaires qui assurent leur pérennité, leur paternité par un identifiant unique (type DOI, Handle, ARK...), leurs conditions de (ré)utilisation sous licence. Les données sont autonomes, leur normalisation et leur standardisation leur apportent visibilité et interopérabilité dans la perspective de leur réutilisation.

L'ouverture des données nous conduit à envisager la recherche à une échelle jusqu'alors inatteignable. Elle donne accès à un réservoir de données en progression constante qui change notre façon de concevoir la recherche. La question de la fouille de textes et de données, rendue possible et efficiente aujourd'hui, représente une avancée majeure, 
même si elle reste cependant complexe à l'échelle nationale, bien que l'exception au droit d'auteur, adoptée en octobre 2016, l'y autorise. D'autres freins doivent être levés, en supprimant par exemple les APC (Articles Processing Charges), en incitant à l'adoption des principes FAIR (facilement trouvables, accessibles, interopérables et réutilisables ${ }^{10}$ ) pour le partage des données sur le Web. Tous les acteurs sont ainsi concernés, du législateur aux acteurs de la recherche, des organismes financeurs aux décideurs politiques, des éditeurs aux porteurs d'infrastructures.

L'arsenal numérique disponible permet en effet d'envisager un croisement de données de manière exponentielle, ouvrant la voie à des développements scientifiques inédits. Les revues, outils majeurs de diffusion de la recherche, participent au premier plan à ce mouvement d'ouverture. Toutefois, tout en se devant d'adopter des principes éditoriaux en adéquation avec le mouvement du libre accès, elles ne peuvent plus se considérer comme un objet en soi, mais doivent s'inscrire dans une chaîne élargie de gestion et de diffusion de la recherche, "comme un espace en interconnexion" (Langlais $2016: 11$ ).

\section{Cinq revues d'archéologie harmonisent leur politique d'édition numérique}

C'est dans ce contexte national favorable que, parmi la cinquantaine de revues françaises consacrées à l'archéologie ${ }^{11}$, cinq d'entre elles ont souhaité, dès 2017 (Allainguillaume et al. 2017), se regrouper pour organiser ensemble la modernisation de leur chaîne de production éditoriale et le choix d'outils orientés Web. L'association repose sur la volonté des éditeurs et des spécialistes de l'information d'utiliser opportunément les outils spécialisés à leur disposition ${ }^{12}$. La décision commune a certainement été favorisée par plusieurs éléments :

- l’implication des éditeurs dans le réseau Médici, observatoire de l'évolution des métiers de l'édition scientifique publique et lieu de veille sur de nouvelles formes numériques d'édition

- la présence de certaines de ces revues au sein de maisons des sciences de l'homme (MSH) : les titres de Gallia-Archéologie des Gaules et Gallia Préhistoire à la MSH Mondes (Maison archéologie \& ethnologie René-Ginouvès, ou MAE, jusqu'en 2020) à Nanterre, $A r$ chéologie médiévale au Centre Michel de Boüard - Centre de recherches archéologiques et historiques anciennes et médiévales (CRAHAM), Préhistoires méditerranéennes ainsi que d'autres titres à la Maison méditerranéenne des sciences de l'homme (MMSH) à Aixen-Provence où se crée un pôle éditorial orienté vers l'archéologie

- la mise en place de la chaîne de production éditoriale Métopes ${ }^{13}$ (Méthodes et outils pour l'édition structurée ; voir infra), portée par le pôle «Document numérique » de la Maison de la recherche en sciences humaines (MRSH) de Caen

- la mise à disposition, pour tous les acteurs de l'archéologie, du thésaurus PACTOLS de Frantiq ${ }^{14}$ (Fédération et ressources sur l'Antiquité - Groupement de services du CNRS), initialement conçu pour les bibliothèques 
- la prise en compte de ce thésaurus pour l'archéologie par les acteurs nationaux de l'édition scientifique : Métopes et OpenEdition

\section{Les revues impliquées dans le projet}

D'audience nationale et internationale, elles représentent un panel exemplaire, par l'espace géographique couvert et les domaines de spécialité traités, de la variété des modèles éditoriaux et de diffusion en place. Elles ont toutes une histoire longue (depuis les années 1940 et 1950 pour Gallia - Archéologie des Gaules et Gallia Préhistoire) et ont connu des évolutions non linéaires, liées à la fois à la conjoncture économique défavorable à la stabilité des équipes, à l'internationalisation de la publication scientifique qui sollicite des contenus multilingues et à la difficulté à aborder le tournant du numérique. Elles bénéficient du soutien de l'INSHS du CNRS.

- Archéologie de la France-Informations (AdlFI) (e-ISSN 2114-0502). À la suite de la section "Informations » de la revue Gallia (1943-1986), puis de la revue Gallia Informations (1987-2001 ${ }^{15}$ ), AdlFI publie depuis 2002 des notices scientifiques pour l'ensemble des opérations archéologiques menées en France, en collaboration avec les services régionaux de l'archéologie (direction régionale des Affaires culturelles). Sa parution est continue et exclusivement numérique. Élaborée en partenariat avec le ministère de la Culture (sous-direction de l'archéologie) et le CNRS (INSHS), elle est hébergée au pôle éditorial de la Maison archéologie \& ethnologie René-Ginouvès à Nanterre. Elle est en ligne sur OpenEdition Journals ${ }^{16}$ depuis 2009.

- Archéologie médiévale (ISSN 0153-9337 ; e-ISSN 2608-4228). La revue associe dans chaque volume annuel des articles de synthèse et une chronique des recherches archéologiques sur les périodes médiévales et modernes menées sur le territoire national et international. En complément de la livraison annuelle, Archéologie médiévale édite des dossiers thématiques alimentés au fil de l'eau, en ligne dans un premier temps et imprimés à la clôture du dossier. Revue préparée entièrement au CRAHAM (UMR 6273, Centre de recherches archéologiques et historiques anciennes et médiévales à Caen) depuis 1971, Archéologie médiévale est éditée par CNRS Éditions. Elle est en ligne sur Persée ${ }^{17}$ - 1971-1999 - depuis 2019 et sur OpenEdition Journals ${ }^{18}$ depuis avril 2018.

- Gallia - Archéologie des Gaules (ISSN o016-4119 ; e-ISSN 2109-9588). La revue publie, annuellement, des dossiers et des articles de synthèse sur les découvertes et les recherches archéologiques en France, depuis la Protohistoire et le premier âge du Fer, l'Antiquité et l'Antiquité tardive jusqu'à la fin des royaumes mérovingiens. Créée par le CNRS en 1943, elle est hébergée au pôle éditorial de la Maison Archéologie \& Ethnologie René-Ginouvès à Nanterre et éditée par CNRS Éditions. Elle est en ligne sur Persée ${ }^{19}$ - 1943-2009depuis 2009 et sur OpenEdition Journals ${ }^{20}$ depuis décembre 2018.

- Gallia Préhistoire (ISSN 016-4127 ; e-ISSN 2109-9642). La revue publie en un volume annuel des articles de synthèse sur les découvertes et les recherches dans le domaine de la Préhistoire en France, depuis le Paléolithique inférieur jusqu'à la fin de l'âge du Bronze. Créée par 
le CNRS, la revue est hébergée au pôle éditorial de la Maison Archéologie \& Ethnologie René-Ginouvès à Nanterre et est éditée par CNRS Éditions. Elle est en ligne sur Persée ${ }^{21}$ - 1958-2014 - depuis 2009, et sur OpenEdition Journals ${ }^{22}$ depuis 2017.

- Préhistoires méditerranéennes (ISSN 1167-492X ; e-ISSN 2105-2565). À la suite de la revue Préhistoire Anthropologie Méditerranéennes (PAM), fondée en 1982, la revue publie depuis 2009 au fil de l'eau et en numérique (publication papier différée), toute contribution originale ayant pour sujet la préhistoire des espaces méditerranéens. Publiée à Aix-en-Provence par l'Association pour la promotion de la préhistoire et de l'anthropologie méditerranéennes (APPAM). Elle est en ligne sur OpenEdition Journals ${ }^{23}$ depuis novembre 2009.

Modèles éditoriaux actuels : parution annuelle et sous forme de dossiers thématiques (Archéologie médiévale et Gallia - Archéologie des Gaules ${ }^{24}$ ), au fil de l'eau pour AdlFI, Préhistoires méditerranéennes et Gallia Préhistoire. Ces deux dernières revues produisent chaque année un volume imprimé différé. Seule AdlFI est uniquement disponible en ligne.

Modes de diffusion : combiné sur Persée et sur OpenEdition Journals (Archéologie médiévale, Gallia - Archéologie des Gaules, Gallia Préhistoire) ou uniquement sur OpenEdition Journals (AdlFI et Préhistoires méditerranéennes). Si toutes ont opté pour le libre accès, trois sont cependant allées plus loin en adoptant l'économie mixte des bouquets Freemium (Archéologie médiévale, Gallia Préhistoire, Gallia - Archéologie des Gaules).

Modes de production : la gestion des flux éditoriaux reste conforme à la tradition académique (réception de l'article par l'éditeur ou l'éditrice, évaluation par les pairs par deux ou trois experts en aveugle, suivi utilisant tableurs ou outils d'aide à la gestion de projets de type Trello) ; la chaîne Métopes est utilisée par les cinq revues.

\section{Leurs objectifs communs}

Regrouper les compétences, travailler en réseau, adopter des outils communs pour favoriser leur interopérabilité et explorer des formes éditoriales nouvelles : tel est le programme que ces cinq revues ambitionnent. La création des pôles éditoriaux dans les maisons des sciences de l'homme de Nanterre et d'Aix-en-Provence témoigne du besoin de rassembler tout autant des compétences métier (maîtrise technique, mise en œuvre de bonnes pratiques...) que des connaissances du contexte disciplinaire spécifique (maîtrise des sujets et des enjeux, des textes techniques, des protocoles de l'environnement...). Ces pôles, ainsi que les éditeurs plus isolés, doivent pouvoir se constituer en réseau pour partager et renforcer les réflexions et les projets afin qu'ils soient portés par tous et puissent servir à tous. La mise en commun de compétences disciplinaires, d'outils et de méthodes est susceptible de favoriser l'adoption et la diffusion de bonnes pratiques éditoriales, tout en rendant plus efficients les échanges avec les auteurs, d'une part, mais également avec les plateformes de diffusion et les éditeurs commerciaux, d'autre part. En outre, il permet de nourrir un échange réciproque, de mobiliser tous ceux qui disposent d'une part d'information, d'imagination ou de savoirfaire pour atteindre des objectifs qui nécessitent une importante capacité de changement. 
Cette harmonisation est en mesure de favoriser, à plus long terme, l'évolution des formes éditoriales de ces revues. Le développement de modèles numériques de structuration des textes et de "sémantisation " des données permet d'envisager d'autres objets éditoriaux, enrichis en métadonnées, par exemple, les modèles de notices archéologiques spécifiques à AdlFI et à Archéologie médiévale. Par ailleurs, il est nécessaire de penser dès à présent à l'intégration des articles de données, absents aujourd'hui du périmètre éditorial de ces revues, pour offrir une passerelle vers des sources essentielles à la lecture avancée des publications en archéologie. La discipline, en effet, produit une masse d'informations que leur format et leurs dimensions rendent encore rarement accessibles, difficilement consultables sur le papier (soumis aux droits des éditeurs pour leur exploitation) et parfois contraintes par les paramètres d'affichage des plateformes en ligne. Données d'observation, données expérimentales et d'analyses, données dérivées et compilées (bases de données, résultats de fouilles de texte ou de données), inventaires et dessins d'objets mobiliers, relevés archéologiques, stratigraphies et plans, images en haute définition et vues tridimensionnelles sont sinon trop rarement accessibles, du moins jamais associés aux publications. Les données doivent être organisées par dépôt pour appuyer la démonstration scientifique et pour favoriser leur usage et leur caractère reproductible. Publication scientifique distincte, mais conjointe à la publication des données de la recherche, le data paper représente ainsi l'un des enjeux majeurs de reconnaissance et d'évaluation de la qualité des travaux scientifiques.

Le numérique offre, par ailleurs, des facilités pour la recherche documentaire que les revues souhaitent valoriser. Les index de chaque revue, établis à partir des mots-clés donnés par les auteurs, sont de fait disparates, à la fois dans leur présentation (le nombre de niveaux d'index varie d'un titre à l'autre) et dans leur valeur lexicale. De plus, les mots-clés des auteurs sont parfois insuffisants, soit trop précis, soit trop vagues, voire inadéquats, pour caractériser objectivement le contenu de l'article. Chaque auteur ayant son propre jeu de mots-clés, il est fréquent de retrouver au niveau de l'index général de la revue des termes similaires sinon synonymes, avec comme corollaire, une consultation peu fiable et malaisée. Il a donc été décidé de s'appuyer sur un vocabulaire unique, contrôlé, qui s'avère indispensable à une recherche efficace, surtout dans la perspective d'une interrogation multi-revues à long terme. L'éditeur doit désormais veiller à la cohérence des métadonnées thématiques qu'il attribue aux contenus, pour l'index homogène de sa revue qu'il faut penser comme partie prenante d'un index élargi aux autres revues.

En poursuivant ce mouvement documentaire, les revues réfléchissent à l'établissement d'une bibliographie numérisée, pensée comme un service adossé à chaque revue. D'abord établies à partir de la compilation des bibliographies des articles de chacun des titres, puis, à terme, fusionnées en un seul réservoir, les références constituent de fait une plusvalue éditoriale pour peu qu'elles soient organisées, normalisées et mises à disposition d'une communauté avide de produits bibliographiques. Cette gestion bibliographique des revues est envisagée de façon partagée avec le logiciel de gestion de références Zotero ${ }^{25}$. La structuration des références bibliographiques stylées dans Métopes facilitera la création d'un fichier de type RIS importable dans Zotero. Parallèlement, 
la bibliothèque partagée sur Zotero constituera un réservoir de notices déjà structurées, exploitable par les auteurs pour la constitution de leur bibliographie et par les éditeurs pour la normalisation et l'enrichissement du réservoir.

\section{Un environnement technologique et disciplinaire particulièrement propice}

L'environnement technique dans lequel se met en place cette harmonisation des pratiques éditoriales numériques est particulièrement favorable. En effet, depuis plusieurs années ${ }^{26}$, le pôle " Document numérique " de la MRSH de Caen met à la disposition des éditeurs publics la chaîne Métopes ${ }^{27}$, outil intégré de production éditoriale exploitant le langage XML ${ }^{28}$. Par ailleurs, la réorganisation du thésaurus pour l'archéologie PACTOLS, développé par la Fédération et ressources sur l'Antiquité (Frantiq), son ouverture à d'autres sphères que celle strictement orientée documentation et bibliothèques ainsi que le développement de ses capacités d'interopérabilité lui confèrent le potentiel nécessaire pour devenir le réservoir de métadonnées thématique central pour l'archéologie. Concernant la bibliographie, le choix s'est porté sur Zotero, déjà largement adopté en archéologie. Logiciel de gestion de références bibliographiques spécialement développé pour les SHS, avec une importante communauté francophone, il permet en outre partage et réutilisation des références. L'association de ces trois outils, interopérables et ouverts, offre donc un cadre idéal à cette coopération.

\section{La chaîne Métopes pour faciliter la production éditoriale}

La chaîne de production éditoriale Métopes concerne le traitement éditorial des textes et leur enrichissement. Sa mise en œuvre s'adapte à tous les environnements informatiques : elle utilise un traitement de texte et un éditeur XML, un jeu de styles et de balises ainsi que des scripts prédéfinis et installés sur le poste informatique de l'éditeur. La prise en main est assurée par les équipes de Numédif (Numérique pour l'édition et la diffusion de la production scientifique) au cours de formations et par le biais d'une assistance à distance. La chaîne Métopes est basée sur le système du SSP (Single Source Publishing), qui permet, à partir d'un fichier-pivot, de produire plusieurs types de supports éditoriaux pour le papier et pour l'électronique. Le manuscrit de l'auteur est stylé avec le traitement de texte puis transformé en fichier XML et enrichi de métadonnées. Le langage XML et la bibliothèque d'encodage TEI, largement exploités par les acteurs de l'édition permettent l'interopérabilité de Métopes avec les plateformes de diffusion déjà en place : grâce aux interconnexions réalisées avec Cairn ou OpenEdition, le fichier pivot s'exporte aisément dans les systèmes de l'un ou de l'autre. De plus, les fichiers sources, textes et illustrations dans leurs différents formats de transformation et de qualités de définition, sont conservés par la revue qui reste ainsi titulaire de son fonds éditorial. 
Numédif s'est lancé dans des expérimentations et des adaptations spécifiques à l'archéologie, qui ont été des éléments déclencheurs de l'adoption de la chaîne Métopes par les cinq revues concernées. Tout d'abord, à la demande et en collaboration avec Archéologie médiévale et AdlFI, un nouveau modèle d'objet éditorial propre aux notices archéologiques a été créé, décrivant de façon synthétique les résultats d'opérations de terrain qui y sont publiés. Les notices présentent l'information selon une organisation systématique qui a facilité le paramétrage d'une structure de données adaptée : intitulé de l'opération, date, site archéologique, institutions responsables de la fouille, périodes historiques, responsable d'opération, etc. Pour ce faire, Numédif a créé un modèle de style propre aux notices archéologiques, en cours de déploiement. Le partenariat avec OpenEdition a permis en parallèle d'ajouter cette " notice archéologique " aux types d'objets éditoriaux reconnus par leur logiciel d'édition électronique Lodel ${ }^{29}$. Ensuite, utilisant les développements du logiciel de gestion de thésaurus Opentheso, Métopes procède à la mise en place dans son interface d'une liaison dynamique avec le thésaurus PACTOLS, facilitant ainsi l'ajout dynamique de métadonnées thématiques, géographiques et chronologiques spécialisées pour l'archéologie. Les éditeurs puisent directement dans le réservoir les concepts utiles à l'indexation de leurs contenus, tant en termes d'articles que de notices. Ils conservent par là même un accès permanent à la dernière mise à jour du thésaurus. En rapatriant non seulement l'intitulé du concept, mais aussi son arborescence et son identifiant pérenne ARK, ils peuvent construire des index à plusieurs niveaux et conservent ainsi un lien avec le référentiel-source.

\section{Le thésaurus PACTOLS pour l'homogénéisation des mots-clés}

L'harmonisation des mots-clés et la possibilité d'effectuer des recherches sur toutes les revues supposent que les métadonnées thématiques proviennent d'une source unique, organisée et contrôlée. Le thésaurus PACTOLS ${ }^{30}$ a été choisi pour de multiples raisons. La première est son adéquation aux contenus scientifiques des revues et sa capacité à évoluer. Élaboré pour permettre l'indexation des publications scientifiques signalées au catalogue collectif de Frantiq, il couvre tous les domaines de l'archéologie depuis la Préhistoire jusqu'à nos jours. Il est multilingue et enrichi en permanence de façon collaborative. Sa normalisation, la citabilité de ses concepts et sa gestion avec le logiciel libre Opentheso le rendent interopérable.

La volonté affirmée de Frantiq de porter le thésaurus sur le Web et de l'ouvrir plus largement à d'autres usages que l'indexation classique de la littérature scientifique a conduit à entreprendre un projet de réorganisation du thésaurus qui doit consolider sa structure et favoriser son appropriation par la communauté scientifique (Nouvel 2018). C'est dans ce cadre que Frantiq accompagne les nouveaux utilisateurs de PACTOLS, les éditeurs en particulier, pour les sensibiliser aux techniques documentaires de l'indexation et pour participer de façon collaborative à son alimentation et à son enrichissement (Nouvel et Rousset 2018). Ensuite, un travail est conduit sur le contenu lexical du thésaurus pour le rapprocher au plus près du vocabulaire utilisé par le chercheur. Il porte essentielle- 
ment sur des enrichissements, par intégration de vocabulaires de spécialités qui complètent certains domaines peu développés du thésaurus, par l'ajout de traductions et la multiplication des relations d'association. Ainsi, plus de 300 termes issus des index de la revue Archéologie médiévale ont été examinés et alignés sur le thésaurus, conduisant à 75 créations et à l'enrichissement d'une centaine de concepts existants (traductions, définitions), avec comme corollaire la modification de certaines hiérarchies. Un travail similaire est en cours pour la revue GalliaArchéologie des Gaules et pour AdlFI qui ont des index aux contenus très proches. À la demande de ces revues et pour les besoins spécifiques de l'indexation des notices archéologiques ayant trait à l'espace français, PACTOLS a étoffé sa liste de lieux. Le fichier des régions et des communes de France a été fourni par l'Institut de l'information scientifique et technique du CNRS (INIST) dans le cadre d'une opération plus vaste de Frantiq pour l'alignement du micro-thésaurus Lieux avec GeoNames. Ce sont ainsi plus de 38 ooo entrées géographiques qui ont été versées dans PACTOLS en 2018, enrichies d'alignements avec plusieurs référentiels nationaux et internationaux ${ }^{31}$, de géolocalisation, de notes diverses, qui remplacent ou complètent les concepts déjà présents. Les besoins des revues sont ainsi couverts et profitent aussi aux autres utilisateurs du thésaurus.

Par ailleurs, le paramétrage de Métopes permettra aux éditeurs de récupérer dans les métadonnées thématiques toutes les informations correspondant aux concepts PACTOLS choisis : intitulé, arborescence, enrichissements, identifiant pérenne ${ }^{32}$. L'utilisation qui est faite de ces données dépend ensuite des capacités des plateformes à les intégrer en tout ou partie. Pour les versions imprimées des revues, seuls les intitulés sont conservés. Pour ce qui concerne les versions électroniques, il est tentant d'envisager le chargement de l'arborescence en vue de construire automatiquement des index hiérarchisés, en particulier pour les index géographiques. De plus, l'exploitation du potentiel offert par les identifiants pérennes pour faciliter l'interconnexion des revues aux autres systèmes utilisant PACTOLS, permettrait a minima l'interrogation interrevues telle qu'elle a été pensée au départ. L'étude de faisabilité menée avec les équipes d'OpenEdition à l'été 2017 a malheureusement montré les contraintes liées à la plateforme de diffusion : si les maquettes ont pu être adaptées à un nouveau style d'affichage, comme cela a été fait pour les notices archéologiques, les évolutions nécessaires du logiciel Lodel ne pouvaient répondre à ces attentes-ci. Début 2019, la prise en compte de ces besoins par OpenEdition a donc abouti à la présentation d'une version rénovée de son moteur autorisant un périmètre de recherche élargi, sans qu'il s'appuie encore sur des vocabulaires liés ${ }^{33}$. 


\section{Situation actuelle et perspectives}

\section{Une harmonisation déjà amorcée}

À ce jour, les cinq revues impliquées dans l'opération d'harmonisation de leur politique numérique ont commencé à réaliser certains de leurs engagements. L'intégration de la chaîne de production éditoriale Métopes est effective dans les cinq revues.

Le rapprochement de leurs index thématique et géographique avec les concepts du thésaurus PACTOLS est en cours. Ce sont $80 \%$ des motsclés d'index qui ont trouvé une équivalence, le reste a suscité la proposition de candidats à ajouter au thésaurus. Celui-ci a ainsi augmenté la part de vocabulaire spécialisé, conformément à son plan de réorganisation et les revues utilisent désormais PACTOLS comme réservoir pour leurs métadonnées thématiques et géographiques. Les revues deviennent des acteurs à part entière de la chaîne de traitement documentaire puisque les mots-clés des articles peuvent être en toute confiance réutilisés pour l'indexation des notices des catalogues de bibliothèques. Avec les projets de gestion des données scientifiques ou de valorisation patrimoniale qui enrichissent les descriptions de leurs contenus avec les concepts PACTOLS ${ }^{34}$, elles intègrent le cercle des nouveaux utilisateurs du thésaurus.

Lors de la phase préparatoire à l'utilisation du thésaurus PACTOLS, les équipes éditoriales ont dû se familiariser aux principes de l'indexation documentaire avec un vocabulaire structuré et contrôlé, élargissant leur champ d'action, tout comme elles ont dû initialement se former aux bases du langage XML-TEI. Ces nouvelles compétences ne remettent pas en question le métier d'éditeur, mais constituent un important changement qui a été présenté, pour la revue Archéologie médiévale, à l'atelier «Dialogu'IST » en novembre 2017 sur l'évolution du travail d'éditeur de revue en archéologie ${ }^{35}$. Les enjeux vont bien au-delà de la publication des résultats de la recherche scientifique. Il s'agit de rendre (rapidement) accessible, dans un souci de transparence et de reproductibilité, tout ce qui concourt à l'avancée de la science.

Afin de préparer au mieux l'indexation lors de la collecte des textes de la chronique des fouilles d'Archéologie médiévale, la revue a mis au point un formulaire de saisie en ligne qui permet aux auteurs de remplir, en lien avec PACTOLS, des champs de mots-clés. Ces mots-clés sont bien sûr contrôlés et enrichis, mais l'utilisation du formulaire facilite le travail de la rédaction sur les 250-300 textes reçus par an.

Par ailleurs, en prévision d'une éventuelle future base de données de notices archéologiques, la revue Archéologie médiévale s'investit dans l'enrichissement en métadonnées des textes de la chronique des fouilles. Notamment, un gros travail de recherche est effectué, en collaboration avec les opérateurs archéologiques, afin de renseigner des liens vers les rapports d'opérations en ligne. L’idée de cette base de données, outre des recherches croisées et avancées, est de devenir un outil précieux pour les chercheurs.

Pour ce qui regarde la revue Gallia-Archéologie des Gaules, l'utilisation de Zotero a été envisagée dans trois directions : 
- Premièrement, établir une base bibliographique Zotero ${ }^{36}$ exhaustive des articles parus dans la revue depuis 1943. La base a été construite afin de permettre un accès direct aux ressources en ligne (fac-similé ou plein texte). Elle permet une interrelation entre articles afin de faciliter la navigation dans le corpus, notamment pour les articles de synthèse publiés sur plusieurs années/volumes. Un pré-encodage des enrichissements typographiques (italiques, capitales et petites capitales) permet un export normalisé des références sélectionnées pour les constitutions de bibliographies. Ce chantier a été mené à bien en 2018 et a servi de socle à la construction d'une collection HAL de la revue, mise en ligne le 9 janvier $2019^{37}$. Gallia-Archéologie des Gaules fournit et garantit ainsi les références fiables de ses propres articles ; elle met à disposition de ses lecteurs une information de première main, en relation dynamique entre HAL, OpenEdition Journals et Persée via une base unique, sans doublons et sans ambiguïtés. Cette base est également mobilisée pour de futures explorations cartographiques par l'identification d'entités géographiques nommées, couplée à un système d'information géographique (SIG). L’objectif est double :

- pour les lecteurs : permettre une future exploration cartographique du corpus des articles publiés (renvoyant vers les publications hébergées en ligne) ;

- pour la revue : disposer d'un outil d'aide à la décision (politique éditoriale, appels à articles) en ciblant des champs géographiques, chronologiques ou thématiques à investir ou à réinvestir.

- Deuxièmement, la revue Gallia - Archéologie des Gaules travaille à la mise à disposition d'une feuille de style Zotero tenant compte de ses recommandations aux auteurs suivant l'exemple des revues Gallia Préhistoire et Archéologie médiévale qui proposent déjà leur propre feuille de style Zotero. Elle permettra une mise en forme automatique des bibliographies d'articles soumis au comité, réduisant ainsi le temps de travail des auteurs, des évaluateurs et des éditeurs.

- Troisièmement, de façon plus prospective, le secrétariat de rédaction de la revue Gallia - Archéologie des Gaules ambitionne de publier une base Zotero de toutes les références publiées dans ses articles depuis une dizaine d'années, afin de réduire le temps de vérification de références récurrentes et d'en optimiser le rendu. 


\section{Des perspectives intégrant infrastructures et plateformes de valorisation des données}

Pour aller jusqu'au bout de la démarche concernant l'adoption du thésaurus PACTOLS, il conviendra qu'OpenEdition autorise l'accès de ses plateformes de revues et de livres aux webservices d'Opentheso qui permettent, en retour du téléchargement des mots-clés, le comptage de leur occurrence dans les réservoirs liés et son affichage dans l'interface de consultation du thésaurus. Si OpenEdition est favorable au principe de ce flux remontant qui va dans le sens de l'ouverture et des échanges d'information, ses modalités de mise en œuvre doivent encore en être définies. Aujourd'hui, seuls les concepts exploités dans le catalogue collectif de Frantiq donnent ces statistiques d'usage. Le système de gestion de bibliothèque, connecté via Opentheso à PACTOLS, renvoie le nombre d'occurrences du concept dans le réservoir bibliographique. L'objectif de Frantiq est que puissent s'afficher ces occurrences pour tous les corpus et réservoirs de données liés. Des connecteurs sont déjà disponibles pour plusieurs systèmes ${ }^{38}$. Ainsi, les utilisateurs de PACTOLS pourront rebondir d'un réservoir de données à l'autre, d'un type d'information à un autre et interroger, à partir d'un concept précis, à la fois des ressources bibliographiques (le catalogue et les revues) et des données (archives scientifiques, systèmes d'enregistrement de terrain, bases de données de recherche). PACTOLS jouera alors pleinement le rôle de référentiel pivot pour l'archéologie que les revues ont bien saisi et auquel elles contribuent déjà.

Si les revues s'engagent d'elles-mêmes dans cette démarche d'ouverture et de collaboration, il en est tout autant des infrastructures de recherche liées à l'édition scientifique. Elles apportent un soutien permanent à la diffusion de bonnes pratiques. Au-delà de la gestion d'un flux éditorial lié à la production des publications (réception, sélection, expertise, préparation de copie, structuration pour édition papier ou électronique des articles), ces infrastructures favorisent la mise en place de workflows intégrés associant publications, métadonnées, données. Cela révèle une évolution des types de contenus à publier, des méthodes et des outils, l'ensemble témoignant de l'évolution du métier d'éditeur. Celui-ci est de plus en plus souvent amené à interagir en cohérence avec son auteur pour préparer l'éditorialisation des données accumulées tout au long du processus scientifique qui mène à la publication. Ainsi, les jeux de données constitués au cours du développement d'une recherche deviennent tout aussi importants que la préparation de la synthèse des résultats scientifiques. Leur mise à disposition à l'issue d'un programme de recherche est même de plus en plus souvent exigée par les partenaires financiers (demandes de financement de projets européens H2O2O, programmes ANR). Il s'agit d'en assurer la qualité par l'évaluation par les pairs (au même titre que tout article scientifique), d'en assurer la paternité, la visibilité, la pérennité, les conditions de réutilisation.

Plusieurs plateformes en cours de déploiement entendent accompagner ce changement de paradigme.

La plateforme OpenArchaeo ${ }^{39}$, développée par l'équipe LAT (Laboratoire archéologie et territoires) de l'UMR Citeres (Cités, territoires, environnment et sociétés ${ }^{40}$ ) à Tours, souhaite mettre l'accent sur 
l'interopérabilité des données archéologiques. L'objectif de cette plateforme est double. Il s'agit, d'une part, de mettre à disposition sur le Web sémantique les jeux de données archéologiques produits dans le cadre du consortium MASA ${ }^{41}$ labellisé par la TGIR Huma-Num, alignés avec l'ontologie du CIDOC $\mathrm{CRM}^{42}$ et ses extensions dédiées à l'archéologie. Il s'agit d'autre part de proposer une interface d'interrogation intuitive pour les données archéologiques inspirée du moteur de recherche ResearchSpace ${ }^{43}$ mis en place par le British Museum, OpenArchaeo étant pour sa part spécifiquement dédié à l'archéologie.

De son côté, la plateforme ArcaDIIS (Archaeological Data Infrastructure for Interoperability and Sharing), développée par le Laboratoire méditerranéen de Préhistoire Europe Afrique (Lampea ${ }^{44}$ ), à Aix-en-Provence) entend rendre visibles et évaluables les données de l'archéologie et des sciences associées (environnement, vie et santé). Cette plateforme collaborative, évolutive et modulaire s'inscrit dans une démarche de science ouverte et exploite les standards du Web sémantique (Delaforge 2016, Renault et Nouvel 2018). ArcaDIIS accompagne les bonnes pratiques éditoriales mises en place par les revues d'archéologie et complète les solutions numériques par un accès aux données de la recherche et la production de formats de publication spécifiques. Elle propose l'interconnexion des différentes ressources qui accompagnent tout projet de recherche en archéologie, dans les sciences et domaines connexes, de son élaboration à la production de ses résultats et de leur évaluation. ArcaDIIS est ainsi conçue comme un hub éditorial qui intègre données, data papers et littérature grise des projets de recherche. Elle repose sur la structuration et la qualification des jeux de données avec les référentiels de la discipline préalablement à leur dépôt. Elle en assure la paternité par l'attribution d'identifiants pérennes et, en réutilisant les métadonnées, systématise la production d'articles de données versés contractuellement en archives ouvertes. La structuration du data paper, en collaboration avec Métopes, et son processus d'évaluation sont actuellement mis en place par les équipes éditoriales des revues concernées sous l'impulsion de Préhistoires méditerranéennes.

\section{Conclusion}

L'adoption de processus éditoriaux vertueux comme ceux décrits au cours de cet article peut contribuer à consolider l'organisation structurelle de la publication française en archéologie. Cette transformation dans l'organisation des processus éditoriaux représente une évolution cohérente en phase avec les services et structures soutenus par les institutions qui les portent.

Ce modèle, articulé autour de l'exploitation de la chaîne Métopes et du thésaurus PACTOLS, incite à réfléchir à l'exploitation par les revues de la discipline du potentiel des outils basés sur les standards du Web, tels le langage XML et ses différents dérivés : TEI, pour l'enrichissement des textes, SKOS pour l'utilisation des vocabulaires de référence contrôlés en ligne, etc. En cohérence avec les politiques éditoriales, la maîtrise de cette chaîne permet aux revues de contrôler leur fonds éditorial puisque la validation de l'article s'effectue à cette étape, indépendamment de la forme finale de l'article, électronique ou maquettée pour l'impression.

Néanmoins, il semble nécessaire de développer des modèles édito- 
riaux complémentaires associant notamment les données de la recherche en archéologie et sciences connexes qui prendraient en compte cet élargissement des besoins en exploitant les standards ouverts et interopérables pour une meilleure visibilité des travaux relevant de leur compétence.

Les missions et les métiers de l'éditeur évoluent, la manière de publier la science évolue. Au-delà de l'aspect purement formel et technique, la structuration des textes (et des données) amène à un travail d'analyse et de perception des sources en lien avec les connaissances de la discipline. L'utilisation d'outils issus de la sphère documentaire est un témoignage plus que manifeste des compétences que l'éditeur en archéologie met en œuvre pour épouser au plus près les évolutions de son champ disciplinaire.

Ce travail autour des cinq revues d'archéologie de ce projet vise à terme à élargir le modèle créé et à le proposer aux autres revues d'archéologie nationales ou interrégionales. La recherche en archéologie aurait beaucoup à gagner à disposer de données interopérables et consultables à grande échelle.

\section{Bibliographie}

Allainguillaume, Micaël, Marie-Adèle Turkovics, Astrid Aschehoug, Nicolas Coquet, Blandine Nouvel et Stéphane Renault. 2017. "Vers une harmonisation numérique des revues d'archéologie ». Poster présenté aux $7^{e s}$ Journées Médici, Dijon, 9-11 octobre. https://halshs. archives-ouvertes.fr/halshs-01614014/document.

Archambault, Éric et Étienne Vignola-Gagné. 2004. «L'utilisation de la bibliométrie dans les sciences sociales et les humanités». Rapport final Science-Metrix. Montréal : Conseil de recherches en sciences humaines du Canada (CRSH). http://www.science-metrix.com/pdf/SM_2004_008_CRSH_Bibliometrie_Sciences_Sociales.pdf.

Aubin, Gérard et Comité des publications et de la diffusion de la recherche archéologique (CPDRA). 2007. "Enquête sur les revues d'archéologie du territoire national ». Paris : Ministère de la Culture et de la Communication. http://www.culture.gouv.fr/content/download/64668/493867/file/enquete_revues_archeo_2007.pdf.

Barthonnat, Céline, Cécile Beauchamps, Odile Contat, Anne-Laure Stérin et Céline Vautrin. 2019. "Recommandations et aspects juridiques relatifs à la création et à la diffusion d'une revue scientifique ». Paris : Bibliothèque scientifique numérique (BSN). https://hal.a rchives-ouvertes.fr/hal-01960919/document.

Boucharlat, Élise et Bernard Mandy. 2014. "Enquête sur les revues d'archéologie du territoire national : les revues interrégionales : 2007-2012. Complément à l'enquête publiée en 2007 ». Paris : Ministère de la Culture et de la Communication. http://www.culture.gouv.fr/Media/Thematiques/Archeologie/Fichier-PDF/etude-rapport/2014_enquete_re-

vue_archeo_complement.

Contat, Odile et Didier Torny. 2015. « Les revues en sciences humaines et sociales à l'heure des communs ». Revue d'histoire moderne \& contemporaine 62-4 bis (5) : 62-70.

Daunois, Thierry et Clotilde Roussel. 2012. « Fonctions et métiers de l'édition scientifique publique. Résultats de l'étude menée par le groupe de travail "Bonnes pratiques : nomenclature métiers du réseau Médici” ". Poster présenté aux $3^{\text {es }}$ journées $d u$ réseau Médici, Caen, 3-4 avril. https://hal.archives-ouvertes.fr/hal-02051973.

Delaforge, Nicolas. 2016. «Mnemotix : coopérative en ingénierie des connaissances et des données ». Communication présentée à la journée d'étude Le Web sémantique pour les données culturelles, Paris, 12 décembre. http://www.culture.gouv.fr/Thematiques/Innovationnumerique/Donnees-publiques/Le-Web-semantique-pour-les-donnees-culturelles-Journ ee-d-etudes-Lundi-12-decembre-2016.

Guédon, Jean-Claude. 2014. "Le libre accès et la "Grande Conversation" scientifique ". Dans Pratiques de lédition numérique, édité par Marcello Vitali-Rosati et Michael E. Sinatra, 111-126. Montréal : Presses de l'université de Montréal. http://www.parcoursnumeriques-pum.ca/le-libre-acces-et-la-grande-conversation-scientifique. 
Henny, Jean-Michel. 2015. L'Édition scientifique institutionnelle en France : état des lieux, matière à réflexions, recommandations. Paris : AEDRES (Association des éditeurs de la recherche et de l'enseignement supérieur). https:/www.enssib.fr/bibliotheque-numerique/documents/65757-l-edition-scientifique-institutionnelle-en-france.pdf.

Hoareau-Dodinau, Jacqueline. 2015. « Avant-propos ». Dans L'Édition scientifique institutionnelle en France : état des lieux, matière à réflexions, recommandations, édité par JeanPierre Henny. Paris : AEDRES (Association des éditeurs de la recherche et de l'enseignement supérieur). https://www.enssib.fr/bibliotheque-numerique/documents/65757-l-edition-scientifique-institutionnelle-en-france.pdf.

Langlais, Pierre-Carl. 2016. "Étude critique des nouveaux modes d'éditorialisation” de revues scientifiques en accès-ouvert ». Paris : Bibliothèque scientifique numérique. https:/ /hal.archives-ouvertes.fr/hal-01388556/document.

Moore, Samuel A. 2017. «A Genealogy of Open Access : Negotiations between Openness and Access to Research ". Revue française des sciences de l'information et de la communication 11 (août). https://doi.org/10.4000/rfsic.3220.

Nouvel, Blandine. 2018. "Évolution et nouvelles pratiques autour du thésaurus PACTOLS de Frantiq pour l'édition numérique en archéologie ". Communication présentée à la $10^{e}$ édition des FréDoc : Démarches innovantes en IST : expérimenter, proposer, (se) réinventer, Albi, 3-5 octobre. https://fredoc2018.sciencesconf.org/data/pages/PACTOLS_B_Nouvel.pdf.

Nouvel, Blandine et Miled Rousset. 2018. "Produire et diffuser des métadonnées thématiques pour l'archéologie : entre savoir-FAIR et pratique collaborative ». Communication présentée à la journée thématique Interopérabilité et pérennisation des données de la recherche : comment FAIR en pratique ? Paris, 27 novembre. https://webcast.in2p3.fr/video/produire-et-diffuser-des-metadonnees-thematiques-pour-larcheologie-entre-savoir -fair-et-pratique-collaborative.

Renault, Stéphane et Blandine Nouvel. 2018. « ArcaDIIS (Archaeological Data Infrastructure for Interoperability and Sharing). Rendre visibles et évaluables les données de l'archéologie. " Poster présenté aux Premières journées nationales de la science ouverte : De la stratégie à l'action. Paris, 4-6 décembre. https://jnso2018.sciencesconf.org/data/pages/ArcaDIIS. pdf.

Suber, Peter. 2016. Qu'est-ce que l'accès ouvert? Marseille : OpenEdition Press. http://books. openedition.org/oep/1605.

\section{Notes}

1 Cet article est le prolongement d'un travail collectif présenté sous la forme d'un poster aux $7^{\text {es }}$ Journées du réseau Médici (9-11 octobre 2017, Dijon), disponible sur HAL SHS : http s://hal.archives-ouvertes.fr/halshs-01614014.

2 La majorité des revues nationales ou interrégionales proposent des collections pour publier des synthèses monographiques, ce qui tend à accroître l'impression de morcellement de la publication archéologique française.

3 https://inshs.cnrs.fr/fr/soutien-aux-revues.

4 La plateforme Revues.org est née en 1999, suivie en 2005 par Persée et Cairn.

5 Pour plus de détails : https://www.openedition.org/14043.

6 http://sciencepress.mnhn.fr/fr/periodiques.

7 Voir par exemple à ce propos, Suber (2016) et Moore (2017).

8 http://jussieucall.org/index-FR.html.

9 Pour plus de détail : https://www.ouvrirlascience.fr/plan-national-pour-la-science-ouv erte.

10 Ces principes sont développés dans l'initiative GO FAIR, dont l'objectif est d'ouvrir progressivement les données de la recherche existantes au sein des institutions scientifiques et académiques dans tous les domaines de la recherche et au-delà des frontières nationales : https://www.go-fair.org/fair-principles.

11 Cinquante-deux revues sont recensées dans le domaine "Archéologie (mondes anciens) " sur Persée, quarante-quatre en « Archéologie et études anciennes " parmi les revues de OpenEdition. 
12 Cette harmonisation concerne également la structure des équipes éditoriales qui s'efforcent de suivre les recommandations de l'INSHS pour le cadre légal et les aspects juridiques (Barthonnat et al. 2019) ainsi que les critères Latindex (https://www.latindex.org/la tindex/inicio).

13 L'infrastructure de recherche (IR) Numédif, au sein de laquelle le projet Métopes s'est développé est devenue en 2018 l'IR Métopes : http://www.metopes.fr/.

14 Le thésaurus est organisé en 6 domaines et une liste, dont les intitulés lui ont donné son nom : Peuples et cultures, Anthroponymes, Chronologie, Toponymes, Euvres, Lieux et Sujets. Plus de détails sur : http://frantiq.fr.

15 D’abord publiée sur papier, la revue est transférée sur support numérique en 1997 (CDRom).

16 Le site de la revue : http://journals.openedition.org/adlfi.

17 https://www.persee.fr/collection/arcme.

18 Le site de la revue : https://journals.openedition.org/archeomed.

19 http://www.persee.fr/collection/galia.

20 Le site de la revue : https://journals.openedition.org/gallia.

$21 \mathrm{http}: / /$ www.persee.fr/collection/galip.

22 Le site de la revue : http://journals.openedition.org/galliap.

23 Le site de la revue : http://journals.openedition.org/pm.

24 Gallia-Archéologie des Gaules vient d'adopter en 2019 le fil de l'eau pour ses varia.

25 https://www.zotero.org.

26 Métopes en tant qu'outil existe depuis 10 ans (1999).

$27 \mathrm{http} / /$ www.unicaen.fr/recherche/mrsh/document_numerique et http://www.numedif.fr/metopes.html.

28 Le langage de balisage XML facilite l'échange d'informations sur Internet : https://fr.wikipedia.org/wiki/Extensible_Markup_Language.

29 https://fr.wikipedia.org/wiki/Lodel.

$30 \mathrm{https://pactols.frantiq.fr/opentheso.}$

31 L'alignement des concepts du micro-thésaurus Lieux de PACTOLS s'est fait avec DBpedia, Wikipédia, Wikidata, GeoNames, l'INSEE. Les termes ont été enrichis de définitions et des traductions.

32 Les identifiants pérennes de PACTOLS donnent à chacun de ses concepts le statut d'un objet Web citable. Ils sont de type ARK (Archival Resource Key).

33 Pour en savoir plus sur le nouveau moteur de recherche d'OpenEdition : https://leo.hypotheses.org/15304 et https://search.openedition.org/?s=.

34. On citera par exemple AERBA (Atlas des établissements ruraux de la Beauce antique), Corea (Corpus et ressources archéologiques du Centre Camille Jullian), IMAGE (Itinéraire médiéval des sites archéologiques du Grand Est), Outagr (Inventaire de l'outillage agricole galloromain), RITA (Recherche en images : Tours archéologique).

35 Les ateliers Dialogu'IST (http://renatis.cnrs.fr/spip.php?article361) sont organisés par Renatis (Réseau national des professionnels de l'information scientifique ; http://renatis.cnrs.fr) et visent à réfléchir sur les enjeux de l'information scientifique et technique (IST).

36 https://www.zotero.org/groups/1959376/revues_gallia.

37 https://hal.archives-ouvertes.fr/GALLIA.

38 Koha, gestionnaire de catalogue de bibliothèque ; Atom, gestionnaire d'archives ; Drupal, gestionnaire de contenus en ligne ; Omeka $S$, pour des bibliothèques numériques et des contenus numérisés ; Métopes, pour la production éditoriale.

39 OpenArchaeo est un des outils développés au sein du consortium MASA (Mémoires des archéologues et des sites archéologiques) et proposé à toute la communauté : https:// masa.hypotheses.org/openarchaeo.

40 http://citeres.univ-tours.fr.

41 https://masa.hypotheses.org. 
42 Il s'agit d'un modèle conceptuel propre au patrimoine culturel (ISO21127) qui propose plusieurs modules dont un spécifique à l'archéologie : http://www.cidoc-crm.org.

43 https://public.researchspace.org/resource/Start.

44 http://lampea.cnrs.fr et http://lampea.cnrs.fr/spip.php?article3671.

\section{Auteurs}

\section{Stéphane Renault}

UMR 7269 Lampea, CNRS, Aix-en-Provence, France

Stéphane Renault est archéologue et responsable éditorial (revue Préhistoires méditerranéennes, Collection "Préhistoires de la Méditerranée ») au Laboratoire méditerranéen de préhistoire Europe-Afrique (UMR 7269 Lampea). Il est responsable du réseau national Médici. Il porte le projet de hub éditorial ArcaDIIS pour le partage et la sémantisation des données de l'archéologie et promeut des formes éditoriales spécifiques (data paper).

renault@mmsh.univ-aix.fr

\section{Blandine Nouvel}

UMR 7299 CCJ, CNRS, Aix-en-Provence, France

Blandine Nouvel est archéologue et documentaliste, chargée de la valorisation numérique des productions scientifiques et éditoriales au Centre Camille Jullian (UMR 7299 CCJ). Elle pilote le programme de réorganisation du thésaurus PACTOLS de la Fédération et ressources sur l'Antiquité (Frantiq, Groupement de services du CNRS) pour en faire un référentiel national pour l'archéologie.

nouvel@mmsh.univ-aix.fr

\section{Micaël Allainguillaume}

UMR 6273 CRAHAM, CNRS, Caen, France

Micaël Allainguillaume est responsable éditorial du service revues et communication du Centre Michel de Boüard - Centre de recherches archéologiques et historiques anciennes et médiévales (UMR 6273 CRAHAM). Il est éditeur des revues Archéologie médiévale (CNRS Éditions) et Tabularia (Presses universitaires de Caen) et responsable du projet Archéomed, base de données de notices archéologiques médiévales.

micael.allainguillaume@cnrs.fr

\section{Astrid Aschehoug}

USR 3225 MSH Mondes (MAE René-Ginouvès jusqu'en 2020), CNRS, Nanterre, France Astrid Aschehoug est coordinatrice du pôle éditorial de la Maison archéologie \& ethnologie René-Ginouvès (USR 3225). Elle y assure en partie le secrétariat de rédaction de la revue Gallia - Archéologie des Gaules. Engagée dans la promotion de l'accès ouvert, elle est membre de l'équipe éditoriale de l'épirevue en cours de création Arche@.

astrid.aschehoug@cnrs.fr

\section{Nicolas Coquet}

USR 3225 MSH Mondes (MAE René-Ginouvès jusqu'en 2020), CNRS, Nanterre, France

Nicolas Coquet, archéologue de formation, est chargé de ressources documentaires pour les revues Gallia et Archéologie de la France Informations au sein du pôle éditorial de la Maison archéologie \& ethnologie René-Ginouvès (USR 3225). En relation avec les infrastructures de recherche Métopes et OpenEdition, il a participé au développement de la notice archéologique et de ses métadonnées spécifiques dans la châ̂ne de production XML-TEI. nicolas.coquet@cnrs.fr

\section{Marie-Adèle Turkovics}

UMR 6273 CRAHAM, université Caen-Normandie, Caen, France

Marie-Adèle Turkovics est éditrice au sein du service revues et communication du Centre Michel de Boüard - Centre de recherches archéologiques et historiques anciennes et médiévales (UMR 6273 CRAHAM). Elle est responsable de la rétro-numérisation de la revue Archéologie médiévale et de son indexation dans le cadre du projet Archéomed, base de données de notices archéologiques médiévales.

marie-adele.turkovics@unicaen.fr 


\section{Droits d'auteur}

(c) (7)

Les contenus de la revue Humanités numériques sont mis à disposition selon les termes de la Licence Creative Commons Attribution 4.0 International. 